\title{
Fractional order Fourier transform as a tool for analyzing multi-element optical system
}

\section{César Torres M., Yezid Torres M.}

César O. Torres M., Yezid Torres M., "Fractional order Fourier transform as a tool for analyzing multi-element optical system," Proc. SPIE 9663, Eighth International Topical Meeting on Education and Training in Optics and Photonics, 96632E (6 October 2003); doi: 10.1117/12.2207466

SPIE Event: Eighth International Topical Meeting on Education and Training in Optics and Photonics, 2003, Tucson, Arizona, United States 


\title{
Fractional order Fourier transform as a tool for analyzing multi-element optical system
}

\author{
César O. Torres M. \\ Universidad Popular del Cesar Laboratorio de Optica e Informática, Valledupar, Colombia. \\ torres.cesar@caramail.com \\ Yezid Torres M. \\ Universidad Industrial de Santander (UIS) Grupo de Optica y Tratamiento de Señales, Bucaramanga, Colombia. \\ ytorres@uis.edu.co
}

\begin{abstract}
The ABCD matrix formalism, the Collins formula and the complex amplitude distributions on two spherical surfaces of given curvature and spacing are adapted to the mathematical expression of fractional order Fourier transform. This result provides a general expression as a tool for analyzing complicated systems involving several lenses and mirrors separated by arbitrary distances; for this class of system it is sufficient to specify the ray transfer matrix and the order of fractional Fourier transform to characterize the system completely.

(C)2002 Optical Society of America

OCIS codes: (050) Diffraction
\end{abstract}

\section{Introduction}

The fractional Fourier transform, which is an extension of the conventional Fourier transform to the fractional order has been introduced into the mathematics literature by Namias[1] in 1980; recently, Mendlovic, Ozaktas and others authors [2-6] introduced a new tool for image analysis in optics; since then, its properties, optical implementation and applications have been studied extensively. An operational definition of fractional Fourier transform in optics and the interpretation of fractional order Fourier transform as the mathematical representation of Fresnel diffraction was stated. Lohmann gave a different definition of the fractional Fourier transform that is based on the Wigner distribution functions. The purpose of this paper is to formulate the fractional order Fourier transform operator; this formula gives the direct relationship between input and output of multi-element optical system.

The study of the ray transfer matrix is particularly useful to simplify the analysis of optical situations; the Collins formula, is a diffraction integral formula for complicated optical system and establishes a bridge between the ray optics and wave optics under paraxial approximation. We show how the combinations of the ray transfer matrix, the Collins formula and the fractional order Fourier transform, result in a new approach suitable for the study of optical structures, where the propagation of light can be viewed as a process of continual fractional Fourier transformation.

\section{Ray transfer matrix}

Under paraxial conditions the properties of rays in optical system can be treated with the elegant formalism of the ray transfer matrix; a paraxial ray in a given cross section of an optical system is characterized by its distance of $\mathrm{x}$ from the optic axis and slope $\mathrm{x}^{\prime}$. If this slope is assumed small, the ray path through any given structure depends on the structure's optical properties, of the structure and on the input conditions. In this situation the relation between the input and output parameters is given for:

Eighth International Topical Meeting on Education and Training in Optics and Photonics,

edited by Barry L. Shoop, Grover Swartzlander Jr., Proc. of SPIE Vol. 9663, 96632E

(C) 2003 SPIE, OSA, ICO · doi: 10.1117/12.2207466

Proc. of SPIE Vol. 9663 96632E-1 


$$
\left(\begin{array}{l}
\mathrm{x}_{2} \\
\mathrm{x}_{2}^{\prime}
\end{array}\right)=\left(\begin{array}{ll}
A & B \\
C & D
\end{array}\right)\left(\begin{array}{l}
\mathrm{x}_{1} \\
\mathrm{x}_{1}^{\prime}
\end{array}\right)
$$

The $A B C D$ matrix is called the ray transfer matrix and generally speaking the determinant is unity.

\section{The fractional Fourier transform and the Collins formula}

From plane $U_{A}(\xi, \eta)$ to $U_{P}(u, v)$, the diffraction field amplitude can be written in Collins diffraction integral equation; the Collins formula in space-domain which gives the relationship between the input complex amplitude $U_{A}(\xi, \eta)$ and the output one $U_{P}(u, v)$ can be rewritten as:

$U_{P}(u, v)=\frac{-\mathrm{i}}{\lambda B} \exp \left(\frac{\mathrm{i} \pi D\left(u^{2}+v^{2}\right)}{\lambda B}\right) \int_{-\infty}^{\infty} \int_{-\infty}^{\infty} \exp \left(\frac{\mathrm{i} \pi A\left(\xi^{2}+\eta^{2}\right)}{\lambda B}\right) \exp \left(\frac{-2 \mathrm{i} \pi(u \xi+v \eta)}{\lambda B}\right) U_{A}(\xi, \eta) d \xi d \eta$

Illuminating the input plane $U_{A}(\xi, \eta)$ with the spherical wave of the radius $\frac{R_{1}}{A}>0$, after a little algebra; the equation (2) can be written in a considerably simpler manner in terms of the fractional order Fourier transform given by:

$U_{P}(u, v)=\frac{2 \pi \sin \alpha}{i \lambda B} \exp \left[\left(\frac{D}{B}-\frac{\cos ^{2} \alpha}{A\left(1-\frac{B}{R_{1}}\right) B}\right) \frac{i \pi\left(u^{2}+v^{2}\right)}{\lambda}\right]\left[\exp \left[-i\left(\frac{\pi}{2}-\alpha\right)\right]\right] \mathfrak{J}^{\alpha}\left[U_{A}(\xi, \eta)\right]$

Where:

$\Im^{\alpha}\left[U_{A}(\xi, \eta)\right]=\frac{\left[\exp \left[i\left(\frac{\pi}{2}-\alpha\right)\right]\right]}{2 \pi \sin \alpha} \exp \left(\frac{-\mathrm{i}}{2 \tan \alpha}\left(u^{2}+v^{2}\right)\right) \int_{-\infty}^{\infty} \int_{-\infty}^{\infty} \exp \left(\frac{-\mathrm{i}}{2 \tan \alpha}\left(\xi^{2}+\eta^{2}\right)\right) \exp \left(\frac{\mathrm{i}(u \xi+v \eta)}{\sin \alpha}\right) U_{A}(\xi, \eta) d \xi d \eta$

A fractional Fourier transform relation $\mathfrak{J}^{\alpha}$ of order $n_{\alpha}$ between the output field complex amplitude $U_{P}(u, v)$ and the input field complex $U_{A}(\xi, \eta)$, can be obtained with $\alpha=\frac{\pi}{2} n_{\alpha}$; ( $\alpha$ real parameter) .

The phase factor it is a quadratic phase factor representing a quadratic approximation to a spherical wave, therefore the field complex amplitude over the output $U_{P}(u, v)$ is over spherical surface with the radius $R_{2}$ and proportional to the Fractional Fourier Transform of order $\alpha$ of the input field complex amplitude $U_{A}(\xi, \eta)$, Where: 
$R_{2}=\frac{-A B\left(1-\frac{B}{R_{1}}\right)}{A D\left(1-\frac{B}{R_{1}}\right)-\cos ^{2} \alpha}$

Then it can be concluded that any $A B C D$ optical system satisfying the relation (5) can implement a fractional order Fourier transform between spherical surfaces with $R_{1}$ and $R_{2}$ radius.

\section{General optics system analyzed as fractional order Fourier transform.}

Equation (5) implies that the condition for a fractional order Fourier transform is that $B \neq 0$; in this situation the field amplitude $U_{P}(u, v)$ represents the fractional order Fourier transform of the field amplitude $U_{A}(\xi, \eta)$, note that, just as with the wave optics operators, the ray transfer matrices should be applied in the sequence in which the structures are encountered if light propagates first through a structure with ray transfer matrix $M_{1}$, then through a structure with ray transfer matrix $M_{2}$, etc, with a final structure having ray transfer matrix $M_{n}$, then the overall ray transfer matrix for the entire system is $M=M_{n} \ldots . M_{2} M_{1}$.

4.1. Fractional order Fourier transform relation between the amplitude distributions of light on two spherical surfaces of given radii and ray transfer matrix.

Note that Eq (5) implies that $R_{1}>B$ and $R_{2}>\frac{B}{D} ; \quad D \neq 0$; then a fractional order Fourier transform relation exist between two spherical surfaces of radii $R_{1}$ and $R_{2}$. We can now write $\mathrm{Eq}(5)$ in the form:

$$
\cos ^{2} \alpha=A\left(1-\frac{B}{R_{1}}\right)\left(D-\frac{B}{R_{2}}\right)
$$

Then it can be concluded that any $A B C D$ optical system satisfying the relation (6) can implement a fractional order Fourier transform, we now discuss the consequences of this equation from five perspectives:

1. A fractional order Fourier transform relation exists between two spherical surfaces of radii $R_{1}$ and $R_{2}$ if and only $0 \leq A\left(1-\frac{B}{R_{1}}\right)\left(D-\frac{B}{R_{2}}\right) \leq 1$.

2. Letting $\alpha=\pi$ (the parity transformation) it is possible to show that $\mathrm{Eq}(6)$ implies the well known imaging condition for multielement optical system.

3. Letting $\alpha=\frac{\pi}{2}$ (the usual Fourier transform) in Eq (6) we see that the complex amplitude distribution of the field in the spherical surfaces of radii $R_{2}$ is the standard Fourier transform of the field on the spherical surface of radii $R_{1}$. 
4. In Eq (6) we can easily find the confinement stability condition for multielement resonator; which coincides with the result obtained by self - consistence method for resonators. We can say that a fractional Fourier transform relation of order $\alpha$ between spherical surfaces of radii $R_{1}$ and $R_{2}$ implies the confinement stability condition for multielement spherical mirror resonator.

5. In accordance with $\mathrm{Eq}$ (3) and $\mathrm{Eq}$ (6) the diffraction integral evaluation of an multielement optical system can be easily carried out in terms of the ABCD matrix.

In the particular situation when $\alpha=\frac{\pi}{2}$ the Eq (6) implies that $0=A\left(1-\frac{B}{R_{1}}\right)\left(D-\frac{B}{R_{2}}\right) ;$ then the matrix element $A=0$ and the result turns out to the usual Fourier transform.

\subsection{Fractional order Fourier transform relation between planar surfaces.}

We have seen that there exist a fractional order Fourier transform relation between two spherical surface; in Eq (6) we now consider that $R_{1} \rightarrow \infty$ and $R_{2} \rightarrow \infty$ (fractional Fourier transform between planar surfaces ) Eq (6) then becomes $\cos ^{2} \alpha=A D$. Letting $\alpha=\pi$ denote the order of transformation occurring from the input plane $U_{A}(\xi, \eta)$ to the output plane $U_{P}(u, v)$, we can write the imaging condition (for an inverted image) as $1=A D$. The usual Fourier transform corresponds to $\alpha=\frac{\pi}{2}$ and $\mathrm{Eq}(6)$ then becomes $0=A D$; evidently when the matrix elements $A$ and $D$ are equal to zero we see that the complex amplitude distribution of the field in the output plane $U_{P}(u, v)$ is the Fourier transform of the field in the input plane $U_{A}(\xi, \eta)$.

4.3. Fractional order Fourier transform operator.

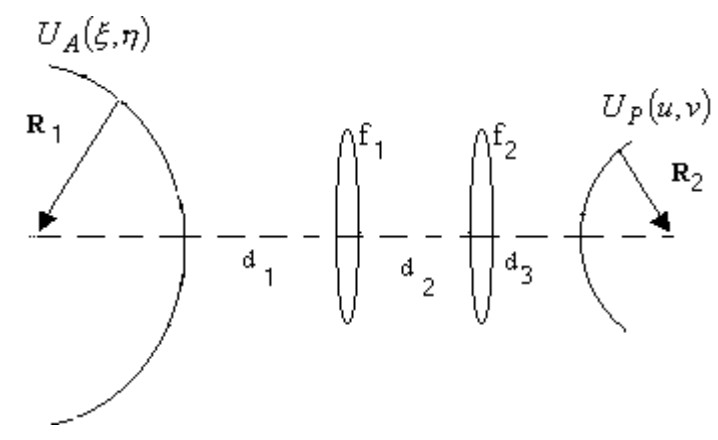

Fig. 1. Optical system between spherical emitter and spherical receiver.

According to equation (3) only one operator is used to express field transfer by diffraction for an optical system described by an $A B C D$ matrix; the relationship between the input and 
output functions can be established by Eq (3). To understand how to use this operator, consider an spherical emitter $U_{A}(\xi, \eta)$ of radii $R_{1}$ followed by section of free space $d_{1}$, followed by lens of focal length $f_{1}$, followed by section of free space $d_{2}$, followed by lens of focal length $f_{2}$, followed by section of free space $d_{3}$, and spherical receiver $U_{P}(u, v)$ of radii $R_{2}$ (Fig. 1). The corresponding $A B C D$ matrix reads as:

$$
\left(\begin{array}{cc}
1-\frac{d_{2}}{f_{1}}+d_{3}\left(-\frac{1}{f_{1}}-\frac{1}{f_{2}}+\frac{d_{2}}{f_{1} f_{2}}\right) & d_{1}+d_{2}-\frac{d_{1} d_{2}}{f_{1}}+d_{3}\left(1-\frac{d_{1}}{f_{1}}-\frac{d_{2}}{f_{2}}+\frac{d_{1}}{f_{2}}+\frac{d_{1} d_{2}}{f_{1} f_{2}}\right) \\
-\frac{1}{f_{1}}-\frac{1}{f_{2}}+\frac{d_{2}}{f_{1} f_{2}} & 1-\frac{d_{1}}{f_{1}}-\frac{d_{2}}{f_{2}}+\frac{d_{1}}{f_{2}}+\frac{d_{1} d_{2}}{f_{1} f_{2}}
\end{array}\right)
$$

According to operator in equation (3), the relationship between the complex amplitude distribution on the spherical emitter $U_{A}(\xi, \eta)$ with radii $R_{1}$ and the complex amplitude distribution on the spherical receiver $U_{P}(u, v)$ with radii $R_{2}$ can be established as:

$U_{P}(u, v)=\frac{2 \pi \sin \alpha}{i \lambda\left(d_{1}+d_{2}-\frac{d_{1} d_{2}}{f_{1}}+d_{3}\left(1-\frac{d_{1}}{f_{1}}-\frac{d_{2}}{f_{2}}+\frac{d_{1}}{f_{2}}+\frac{d_{1} d_{2}}{f_{1} f_{2}}\right)\right)} \exp \left[\frac{i \pi\left(u^{2}+v^{2}\right)}{\lambda\left(R_{2}\right)}\right]\left[\exp \left[-i\left(\frac{\pi}{2}-\alpha\right)\right]\right] \mathfrak{J}^{\alpha}\left[U_{A}(\xi, \eta)\right]$

Given $R_{1}$ and $A B C D$ matrix; if we wished to design a fractional Fourier transform system with specific order $\alpha$ using Eq (5) we can obtain $R_{2}$.

Given $R_{1}, R_{2}$ and $A B C D$ matrix if we wish to design a fractional Fourier transform system using Eq (6) we can obtain the specific order $\alpha$.

In fig 1. alternatively, let us consider an pair of planar surfaces with $R_{1} \rightarrow \infty$ and $R_{2} \rightarrow \infty$, it is now possible derived the well known Fourier transforming properties of lenses by Goodmann $\left(d_{3}=0\right.$ and $\left.f_{2} \rightarrow \infty\right)$, the canonical assemblies by Lohmann, $\left(d_{1}=d_{2} \quad d_{3}=0, f_{1}=f\right.$, and $f_{2} \rightarrow \infty \quad$ type-I setup $) ; \quad\left(d_{1}=d_{3}=0\right.$ and $f_{1}=f_{2}=f \quad$ type-II setup), the imaging condition $\left(\alpha=\pi, d_{3}=0\right.$ and $\left.f_{2} \rightarrow \infty\right)$ optical system as performing two consecutive fractional Fourier transform operations ( $\left.d_{2}=d_{1}+d_{3}\right)$; condition for $U_{P}(u, v)$ to be the coherent image of $U_{A}(\xi, \eta)$ $(\alpha=\pi)$ and $U_{P}(u, v)$ to be the standard Fourier transformation of $U_{A}(\xi, \eta)\left(\alpha=\frac{\pi}{2}\right)$

This result shows that the fractional order Fourier transform operator Eq (3) provide a convenient and systematic technique for analysis of optical system described by an $A B C D$ matrix 


\section{Summary}

In this paper using the Collins formula, the ray transfer matrix and the fractional order Fourier transform we have derived the fractional order Fourier transform operator; in addition this operator provide a new way of analyzing optical system involving several lenses and mirrors separated by arbitrary distances.

\section{Acknowledgments}

The first author would like to acknowledge the Grupo de Optica y Tratamiento de Señales of the Physics School of the Universidad Industrial de Santander (UIS) where the first author carries out studies for obtaining the grade of Ph.D.

\section{References}

1. W. Goodman, “Introduction to Fourier optics," McGraw-Hill, New York, Chap. 3, p. 34, (1996).

2. A. W. Lohmann, "Image rotation, Wigner rotation, and the fractional Fourier transform," J.Opt. Soc.Am. A10, 2181-2186 (1993).

3. S. Granieri, O. Trabocchi and E. E. Sicre, "Fractional Fourier transform applied to spatial filtering in the Fresnel domain," Opt. Comunn. 119, 275-278 (1995).

4. P. Pellat-Finet, "Fresnel diffraction and the fractional-order Fourier transform," Opt. Let. 19, 1388-1390 (1994).

5. H. Ozaktas and D. Mendlovic, "Fractional Fourier transform as a tool for analyzing beam propagation and spherical mirror resonators," Opt. Let. Vol. 19, No. 21, 1678-1680 (1994).

6. H. Ozaktas and D. Mendlovic, "Fractional Fourier optics," J. Opt. Soc. Am. A. Vol. 12, No 4 743-750 (1995) 